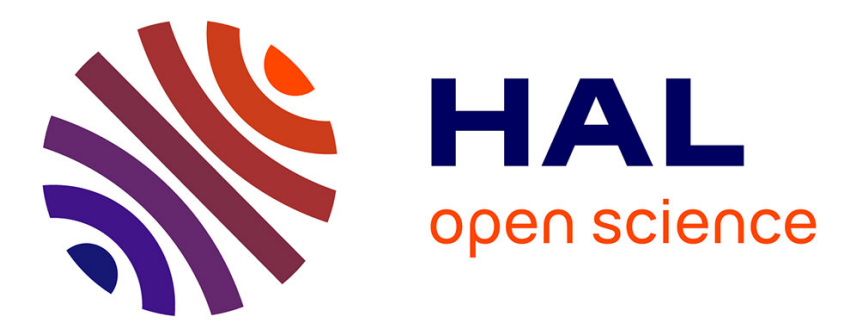

\title{
A periodic inspection and replacement policy for systems subject to competing failure modes due to degradation and traumatic events
}

Khac Tuan Huynh, Anne Barros, Christophe Bérenguer, Inma T. Castro

\section{- To cite this version:}

Khac Tuan Huynh, Anne Barros, Christophe Bérenguer, Inma T. Castro. A periodic inspection and replacement policy for systems subject to competing failure modes due to degradation and traumatic events. Reliability Engineering and System Safety, 2011, 96 (4), pp.497-508. 10.1016/j.ress.2010.12.018 . hal-00790728

\section{HAL Id: hal-00790728 \\ https://hal.science/hal-00790728}

Submitted on 20 Feb 2013

HAL is a multi-disciplinary open access archive for the deposit and dissemination of scientific research documents, whether they are published or not. The documents may come from teaching and research institutions in France or abroad, or from public or private research centers.
L'archive ouverte pluridisciplinaire HAL, est destinée au dépôt et à la diffusion de documents scientifiques de niveau recherche, publiés ou non, émanant des établissements d'enseignement et de recherche français ou étrangers, des laboratoires publics ou privés. 


\title{
A periodic inspection and replacement policy for systems subject to competing failure modes due to degradation and traumatic events
}

\author{
K.T. Huynh ${ }^{\mathrm{a}, *}$, A. Barros ${ }^{\mathrm{a}}$, C. Bérenguer ${ }^{\mathrm{a}}$, I.T. Castro ${ }^{\mathrm{b}}$ \\ ${ }^{a}$ Université de technologie de Troyes - Institut Charles Delaunay and STMR UMR CNRS 6279 - 12, rue Marie Curie, \\ BP2060, 10010 Troyes cedex, France \\ ${ }^{b}$ Departamento de Matemáticas. Escuela Politécnica. 10071 Cáceres, Spain
}

\begin{abstract}
This paper deals with the condition-based maintenance of single-unit systems which are subject to the competing and dependent failures due deterioration and traumatic shock events. The main aim is to provide a model to assess the value of condition monitoring information for the maintenance decision-making. A condition-based periodic inspection/replacement policy is developed and compared with a benchmark timebased block replacement policy.

Numerical results show that it is indeed useful to follow closely the actual evolution of the system to adapt the maintenance decisions to the true system state to improve the performance of maintenance policies. The analysis of the maintenance costs savings can be used to justify or not the choice to implement a policy based on condition monitoring information and to invest in condition monitoring devices.
\end{abstract}

\section{Keywords:}

Gamma process, non-homogeneous Poisson Process, maintenance policy, periodic inspection/replacement, block replacement, dynamic environment

\section{Acronyms}

CBM Condition-based maintenance

TBM Time-based maintenance

B-R Block replacement

P-I/R Periodic inspection/replacement

DTS Degradation-Threshold-Shock

\footnotetext{
* Corresponding author

Email addresses: tuan.huynh@utt.fr (K.T. Huynh), anne.barros@utt.fr (A. Barros), christophe.berenguer@utt.fr (C. Bérenguer), inmatorres@unex.es (I.T. Castro)
} 


\section{Introduction}

Maintenance has an important role directly related to the competitiveness of a company. The maintenance operation not only guarantees the system availability but also improves the system safety and the product quality, and assures the customer satisfaction. Nowadays, the growing dissemination of condition monitoring techniques and of maintenance information management foster the implementation of conditionbased maintenance $(\mathrm{CBM})$ policies among organizations seeking to improve their maintenance performance under budget and resources constraints and to gain a competitive advantage. However, the maintenance decision based on the condition monitoring information returned by inspection on a system can be expensive to implement and an analysis has to be performed to determine whether (and under which conditions) $\mathrm{CBM}$ can be an appropriate choice for an industrial system and whether it can replace with profit a more classical time-based maintenance (TBM) policy [1]. To answer to such a question, it is necessary to perform a comparison between CBM policy and TBM policy under different system characteristics. In the literature, comparison on the performance of CBM policy and TBM policy has been discussed by Mann et al. in [2] and Bouvard et al. in [3]. However, in [2] the comparison is merely a qualitative analysis, while in [3] the quantitative comparison is based on a rather simple degradation model. Moreover even though a lot of maintenance models for deteriorating systems have been proposed in the literature (reviewed by Wang in [4]), both for CBM and TBM strategies, none of them is applicable for a more general deterioration/failure model considered in this paper. Therefore, the main aims of this study are (i) firstly to introduce a an extended deterioration/failure model with dependence for a single-unit deteriorating system; (ii) secondly, to develop a mathematical cost model for a CBM policy (and a TBM policy) for the proposed deterioration/failure model; and (iii) thirdly to show through numerical examples how these models can be used to investigate the value of condition monitoring information and to indicate which type of maintenance policy should be applied according to different conditions and system characteristics.

The degradation behavior of a system is usually modeled by a stochastic process, and developments on maintenance model based on deterioration process have provided satisfactory results in the maintenance operation (see [5,6] for TBM model and [7-9] for CBM model). But, considering a deterioration process only, as in these papers, seems to be unsatisfactory for many application cases to describe the dynamic system characteristics since the system does not only degrade in operating environments, but is also subject to traumatic events or shocks that can lead to a sudden failure [10]. So, we are interested in a more realistic model combining the deterioration process and the shock process. On dealing with competing risk of the degradation process and random shock, most of the present papers assumed that they are independent with each other [11-14], but in many practical situations, the dynamics of the dependent structure between them is of importance and should not be neglected in the competing risk model. Therefore, in this paper, we add a new dimension to the degradation-threshold-shock (DTS) model [12] by introducing the dependence 
between the shock process and the degradation process.

Based on the deterioration/failure model, the mathematical cost models for a CBM policy and a TBM policy are developed. Due to the simplicity and the high usability in practice, the periodic inspection/replacement (P-I/R) policy, whose maintenance decisions are based on the information returned by inspection, is mainly studied in this paper. The block replacement (B-R) policy, whose maintenance decisions are performed regardless of the condition of system, is used as a benchmark TBM policy to assess the value of condition monitoring information in maintenance decision. We do not use age-based replacement policy as a benchmark TBM policy in this work, even if it can be more profitable than B-R policy, as it is not a sensible policy in our case because the system is not replaced immediately upon failure. A comparison of the optimal maintenance cost rate of both P-I/R policy and B-R policy under different conditions and system characteristics will show the advantage and the situation to implement of each maintenance policy.

Hence, the main contributions of the present study are:

1. Expanding DTS model introducing the dependence between the shock process and degradation process.

2. Providing the analytical cost models and performing a quantitative comparison between TBM and CBM based on the more general degradation/failure model.

3. Assessing the value of condition monitoring information for maintenance decision-making.

4. Providing the indicators for choosing the adequate maintenance policy according to different system characteristics.

The remainder of this paper is organized as follows. Section 2 is devoted to model the different competing failures modes of system and to investigate the associated failure times. The detailed description and formulation of both P-I/R policy and B-R policy are represented in section 3. Section 4 deals with analysis and discussions on the sensitivity to different parameters of deterioration/failure model and the maintenance model, and shows the value of condition monitoring information for maintenance decision-making. Finally the paper will be end with some conclusions and some directions for future works.

\section{System degradation and failure modeling}

The present paper considers a single-unit system whose failures are due to the competing causes of degradation and shocks. The system is described by a so-called degradation-threshold-shock (DTS) model [12]. In this model, the deterioration is modeled using a time-dependent stochastic process, and the system is regarded as failed when the degradation process reaches a critical threshold or when a shock occurs although the degradation process has not reached the threshold. As Singpurwalla advocated in [10], such a failure/deterioration model can be seen as a combination and more versatile - and hopefully realistic extension of many classical failure models based either only on deterioration or only on parametric lifetime distributions. More recently, Bocchetti et al. apply this model to describe the competing risks due to wear 
degradation and thermal cracking for the cylinder liners of a marine Diesel engine [15]. In the following, the modeling of different failure modes and the distributions of the associated hitting time is analyzed in more details highlighting the dependence between failure modes.

\subsection{Deterioration-based failure}

\subsubsection{Deterioration modeling}

Consider a system deteriorating with use and age, and subject to continuous accumulation of deterioration. The deterioration evolution of system is usually modeled by a stochastic process. Let $X(t)$ be the accumulated deterioration (or wear level) at time $t$. If no maintenance action is performed, the stochastic process $\{X(t), t \geq 0\}$ is continuous-time and monotonically increasing with $X(0)=0$. The deterioration is strictly increasing which means that the system worsens with time due to ageing and accumulated wear or damage.

Gamma processes were satisfactorily fitted to data of different gradual degradation phenomena such as erosion, corrosion, concrete creep, crack growth, wear of structural components [16-18]. Moreover, the existence of an explicit probability distribution function of gamma process permits feasible mathematical developments. Therefore, since the initial proposal by Abdel-Hameed in [19], the gamma process has been widely studied to apply in maintenance applications by several authors (see [20] for a thorough review on the use of Gamma process in maintenance modeling), and we use it in this study to model the degradation evolution of the system. Note however that an other monotone stochastic process could have been used (e.g. any process from the Levy family) at the price of more difficult mathematical derivations.

Assume that $\{X(t), t \geq 0\}$ is a homogeneous gamma process. It means that the probability density function of deterioration level $X(t)$ at time $t$ is a gamma density function with shape parameter $\alpha t$ and scale parameter $\beta$,

$$
f_{\alpha t, \beta}(u)=\frac{\beta^{\alpha t} u^{\alpha t-1} e^{-\beta u}}{\Gamma(\alpha t)}, \quad u \geq 0,
$$

where $\Gamma$ is the function gamma given by

$$
\Gamma(\alpha)=\int_{0}^{\infty} u^{\alpha-1} e^{-u} d u .
$$

Such a process depends on two parameters $\alpha$ and $\beta$ which allows to model various deterioration behaviors of the system from almost deterministic to highly variable. The parameters $\alpha$ and $\beta$ can be estimated from deterioration data with classical statistical methods. The average deterioration rate is $m=\alpha / \beta$ and its variance is $\sigma^{2}=\alpha / \beta^{2}$.

We assume that, when the level of deterioration exceeds the failure threshold $L$ which depends on the properties of the considered system, a degradation failure happens. We notice that in reality, the failures can be not obvious to the user and cannot be easily characterized and identified. So, the system can be declared as "failed" as soon as a defect or an important deterioration is present, even if the system is still functioning. 
This means that it is no longer able to fulfil its mission in acceptable conditions. In this situation, its high level of deterioration is unacceptable either for economic reasons (poor products quality, high consumption of raw material, etc.) or for safety reasons (high risk of hazardous breakdowns).

\subsubsection{On hitting times of the deterioration process}

To characterize the deterioration-based failures, it is necessary to study the hitting times of the deteriorating gamma process. Two types of hitting times are showed in this section.

Firstly, denoting by $\tau_{L}$ the time at which the degradation failure occurs and by $F_{\tau_{L}}$ its distribution, one obtains

$$
F_{\tau_{L}}(t)=P\left[\tau_{L} \leq t\right]=P[X(t) \geq L]=\int_{L}^{\infty} f_{\alpha t, \beta}(x) d x=\frac{\Gamma(\alpha t, L \beta)}{\Gamma(\alpha t)}, \quad t \geq 0
$$

where

$$
\Gamma(\alpha, x)=\int_{x}^{\infty} z^{\alpha-1} e^{-z} d z
$$

denotes the incomplete gamma function for $x \geq 0$ and $\alpha>0$. Distribution $F_{\tau_{L}}$ in $(2)$ is known as the first hitting time distribution to the level $L$ with density function given by [20]

$$
f_{\tau_{L}}(t)=\frac{\partial}{\partial t} F_{\tau_{L}}(t)=\frac{\alpha}{\Gamma(\alpha t)} \int_{L \beta}^{\infty}\{\log (z)-\psi(\alpha t)\} z^{\alpha t-1} e^{-z} d z, \quad t \geq 0,
$$

where the function $\psi(a)$ is called the digamma function that corresponds to the derivative of the logarithm of the gamma function

$$
\psi(a)=\frac{\Gamma^{\prime}(a)}{\Gamma(a)}=\frac{\partial \log \Gamma(a)}{\partial a} .
$$

Considering another deterioration level $M$ of system with $M<L$ and let $F_{\tau_{M}}$ the time in which, for the first time, the deterioration of the system exceeds the level $M$. In the models showed subsequently, the distribution of the random variable $\tau_{L}-\tau_{M}$ is used but more difficult to derive because of the "overshoot behavior" of the gamma processes [21], i.e., $X\left(\tau_{M}\right) \neq M$ and $\tau_{L}-\tau_{M} \neq \tau_{L-M}$. The distribution of $\tau_{L}-\tau_{M}$ was obtained by [8] and it is given by

$$
\bar{F}_{\tau_{L}-\tau_{M}}(t)=\bar{F}_{\left(\tau_{M}, \tau_{L}\right)}(t, x, y) 1_{\{y>x>0\}}+\bar{F}_{d}(t, x, y) 1_{\{y=x>0\}}
$$

where $1_{\{\}}$denotes the indicator function which equals 1 if the argument is true and 0 otherwise,

$$
\bar{F}_{\left(\tau_{M}, \tau_{L}\right)}(t, x, y)=-\iint_{M<x<L, 0<x+y<L, 0<y}\left(\int_{0}^{\infty} f_{\alpha u, \beta}(x) d u\right) \frac{\partial f_{\alpha t, \beta}(y)}{\partial t} d x d y
$$

and

$$
\bar{F}_{d}(t, x, y)=\int_{t}^{\infty} \int_{0}^{y-t} \alpha \int_{0}^{M} f_{\alpha x, \beta}(w) d w\left(\int_{L-y}^{\infty} \frac{e^{-\beta z}}{z} d z\right) d x d y
$$

Expression (4) is complicated and specially burdensome to compute numerically. This fact makes us propose an approximation of the distribution of the random variable $\tau_{L}-\tau_{M}$ to make the computation faster and 
easier. This approximation is based on the distribution of $\tau_{L-M}$. If the trajectories of the process $\{X(t), t \geq$ $0\}$ were continuous, then $\tau_{L}-\tau_{M}$ would have the same probability as $\tau_{L-M}$. But, since $\{X(t), t \geq 0\}$ is a jump process (see $[20,22]$ for more properties of gamma process), $X\left(\tau_{M}\right) \geq M$ and hence $E\left[X\left(\tau_{M}\right)\right] \geq M$. Bérenguer et al. proposed in [8] as approximation of $E\left[X\left(\tau_{M}\right)\right]$ the following expression

$$
E\left[X\left(\tau_{M}\right)\right]=\frac{\alpha}{\beta} E\left[\tau_{M}\right]=\frac{\alpha}{\beta} \frac{1}{\alpha}\left(\beta M+\frac{1}{2}-\int_{\beta M}^{\infty}(\varphi(y)-1) d y\right),
$$

where $\varphi$ is a function closed to 1 ,

$$
\varphi(y)=\int_{0}^{\infty} f_{u, 1}(y) d u, \quad y \geq 1
$$

hence, when $\beta M$ is large enough (at least greater 1),

$$
E\left[X\left(\tau_{M}\right)\right] \simeq M+\frac{1}{2 \beta}
$$

As a consequence, the distribution of the random variable $\tau_{L}-\tau_{M}$ can be approximated by the distribution of $\tau_{L-M-1 /(2 \beta)}$ when $\beta M<\beta L-1 / 2$, thus the distribution of $\tau_{L}-\tau_{M}$ given in (4) and its density $f_{\tau_{L}-\tau_{M}}$ can be approximated as

$$
\begin{aligned}
F_{\tau_{L}-\tau_{M}}(t) & \simeq F_{\tau_{L-M-\frac{1}{2 \beta}}}(t)=\frac{\Gamma(\alpha t, \beta(L-M)-1 / 2)}{\Gamma(\alpha t)} \\
f_{\tau_{L}-\tau_{M}}(t) & \simeq f_{\tau_{L-M-\frac{1}{2 \beta}}}(t)=\frac{\alpha}{\Gamma(\alpha t)} \int_{(L \beta-M \beta-1 / 2)}^{\infty}\{\log (z)-\psi(\alpha t)\} z^{\alpha t-1} e^{-z} d z,
\end{aligned}
$$

and this approximation will be used later in the numerical examples to compare different maintenance strategies.

\subsection{Shock failure}

\subsubsection{Shock failure modeling}

As mentioned above, in most practical situations the system failure is not solely due to degradation but also is due to traumatic shock events, either internal or external. Moreover the degradation process and shock process can depend each other, for example the higher the degradation, the more the system is vulnerable to the shocks. We assume that the shocks arrival times can be modeled by a nonhomogeneous Poisson process $\{N(t), t \geq 0\}$ with stochastic increasing intensity which depends on the degradation level $r(X(t))$, and that can represented by the following relation

$$
r(X(t))=r_{1}(t) 1_{\left\{X(t) \leq M_{s}\right\}}+r_{2}(t) 1_{\left\{X(t)>M_{s}\right\}}, \quad t \geq 0,
$$

where $1_{\{\}}$denotes the indicator function which equals 1 if the argument is true and 0 otherwise, $r_{1}(t)$ and $r_{2}(t)$ denote two continuous and non-decreasing failure rates at time $t$ with $r_{1}(t) \leq r_{2}(t), \forall t \geq 0$. The quantity $M_{s}<L$ represents a fixed deterioration level. The quantities $r_{1}(t), r_{2}(t)$ and $M_{s}$ are the parameters of shock process and can be estimated form shock failures and deterioration data with classical statistical 
methods. Expression (8) means that the degradation evolution affects to the occurrence of shock failures, that is, the system is more prone to shock failures when the deterioration increases and exceeds a given deterioration level.

\subsubsection{Failure time of the traumatic shock process}

Let $N(t)$ be the number of shock failures in $[0, t]$. Under this model, the failure rate for the shock failures $r(X(t))$ given by (8) is stochastic and the process $\{N(t), t \geq 0\}$ is called a doubly stochastic process or a Cox process (see [23] for a theoretical development of the Cox process). We denote by $\bar{F}_{1}$ and $\bar{F}_{2}$ the survival functions associated with the failure rate functions $r_{1}$ and $r_{2}$, that is,

$$
\left.\bar{F}_{i}(t)=\exp \left\{-\int_{0}^{t} r_{i}(u) d u\right)\right\}, \quad t \geq 0, \quad i=1,2 .
$$

We denote by $\tau_{M_{s}}$ the time that for which the deterioration of the system first exceeds the value $M_{s}$. Let $\tau_{s}$ be the time to an shock failure and $\bar{F}_{s}(t)$ be its survival function, one has

$$
\begin{aligned}
\bar{F}_{s}(t) & =P\left[\tau_{s}>t\right] \\
& =P\left[\tau_{s}>t \mid X(t) \leq M_{s}\right] P\left[X(t) \leq M_{s}\right]+P\left[\tau_{s}>t, X(t)>M_{s}\right] \\
& =\bar{F}_{\tau_{M_{s}}}(t) \bar{F}_{1}(t)+\int_{0}^{t} f_{\tau_{M_{s}}}(u) \bar{F}_{1}(u) \exp \left\{-\int_{u}^{t} r_{2}(v) d v\right\} d u, \quad t \geq 0 .
\end{aligned}
$$

where $F_{\tau_{M_{s}}}$ (resp. $f_{\tau_{M_{s}}}$ ) denotes the distribution (resp. density) of the hitting time for the deterioration level $M_{s}$ obtained using (2) and (3) respectively.

\section{Maintenance models}

This section aims to develop the maintenance models for the deterioration/failure model described in section 2. Due to the simplicity and the high usability in practice, the periodic inspection/replacement (P$\mathrm{I} / \mathrm{R}$ ) policy, whose maintenance decisions are based on the information returned by inspection, is declared as a representative of the class of CBM policies and is mainly studied in this paper. The block replacement (B-R) policy is a "blind" TBM policy used as a benchmark to assess the value of condition monitoring information in maintenance decision. The analytic cost model of each maintenance policy is developed based on the well-known renewal theorem.

\subsection{Assumptions and performance assessment criterion}

\subsubsection{Assumptions}

Consider a non-reparable single-unit system described in section 2. We suppose that the system starts working at time $t=0$. The degradation system of system is hidden and the system failure is non-selfannouncing. This means that the system reveals only its degradation state or its failure through an inspection. These assumptions are reasonable because in practice the system degradation is usually not observed 
directly, and as mentioned above, for the economic and safety reasons the system is considered as "failed" even if it is still running, so no indicator can exhibit the degradation and failure state of system except to do an inspection. Moreover with a system subject to different failure modes, the inspections are really necessary to distinguish between the types of failure which can used for experience feedback and for parameter estimation. The inspections are assumed instantaneous, perfect, non-destructive and incurred a $\operatorname{cost} C_{i}$.

Two maintenance operations are available on the system: the preventive replacement with $\operatorname{cost} C_{p}>C_{i}$ and the corrective replacement with $\operatorname{cost} C_{c}$. A replacement can be either a true physical replacement or an overhaul or repair such that the system is as-good-as-new after the repair. Even though both the preventive and the corrective maintenance actions put the system back in the as-good-as-new state, they are not necessarily identical in practice because the corrective replacement (or renewal) is unplanned and it has to be performed on a more deteriorated system, and the $\operatorname{cost} C_{c}$ can also comprise different costs associated to failure like damage to the environment. It is thus likely to be more complex and more expensive (i.e. $C_{c}>C_{p}$ ). Moreover a replacement, whether preventive or corrective, can be performed only at the discrete times (inspection time in P-I/R policy or regular replacement time in B-R policy), and takes negligible time. Therefore, there exists a system inactivity after failure and an additional cost is incurred from the failure time until the next replacement time at a cost rate $C_{d}$.

\subsubsection{Policy performance assessment criterion}

To evaluate the performance of a maintenance policy, one can use the availability of the maintained system or the overall maintenance cost balance [24]. In this work, we focus only on an asymptotic evaluation of a cost criterion which is the expected maintenance cost per unit over an infinite time span, because this criterion is most used in reality. Under the assumption of an as-good-as-new maintained system, an analytical formula of this long run expected cost rate can be computed thanks to the regenerative properties of the maintained system state [25]

$$
C^{\infty}=\lim _{t \rightarrow \infty} \frac{C(t)}{t}=\frac{E\left[C\left(S_{1}\right)\right]}{E\left[S_{1}\right]}
$$

where $C(t)$ is the cumulated maintenance cost at time $t, S_{1}$ is the length of the first renewal cycle.

\subsection{Periodic inspection/replacement model}

\subsubsection{Periodic inspection/replacement policy}

For this maintenance policy, the system is periodically inspected with period $T$ and with $\operatorname{cost} C_{i}$. At each inspection $T_{i}$ where $i=1,2,3 \ldots$, if the observed degradation level $X\left(T_{i}\right)$ exceeds a threshold $M$ and if no failure occurred, a preventive replacement is performed with cost $C_{p}$. But upon inspection, if the system is detected to be in failure state (either due to degradation $X\left(T_{i}\right) \geq L$ or due to shocks), it is correctively replaced with cost $C_{c}$. In this case, because of the system inactivity after failure, an additional cost is incurred from the failure time until the inspection time at a cost rate $C_{d}$. Practical conditions define the 
constraints of the maintenance costs as follows: $C_{i}<C_{p}<C_{c}$. The inter-inspection interval $T$ and the preventive threshold $M$ are the decision variables of this policy. We seek the optimal values of the decision variables $\left(T_{o p t}, M_{o p t}\right)$ which minimize the cost criterion (11).

\subsubsection{Cost model formulation}

Applying (11) the expected cost rate for this inspection strategy is given by

$$
C_{i n s}(T, M)=\frac{C_{p} P_{p}(T, M)+C_{c}\left(1-P_{p}(T, M)\right)+C_{d} E\left[W_{r}\right]+C_{i} E\left[N_{i}\right]}{E\left[\tau_{r}\right]},
$$

where $E\left[\tau_{r}\right], P_{p}(T, M), E\left[W_{r}\right]$ and $E\left[N_{i}\right]$ are respectively the expected time to a system replacement, the probability of a preventive replacement in a replacement cycle, the expected down time of the system in a replacement cycle, and the expected number of inspections during a replacement cycle. The remaining of this section aims to introduce the analytical formulas of these quantities.

Consider a replacement cycle defined as the time interval between successive replacements of the system. Let $\tau_{r}$ be the time to the replacement of the system. Under the assumptions of the model, $\tau_{r}$ can be given by

$$
\tau_{r}=(k+1) T, \quad k=0,1,2, \ldots
$$

if one of the three following exclusive events occurs

$$
\left\{\begin{array}{l}
k T<\tau_{M}<(k+1) T, N((k+1) T)=0 \\
(k+1) T<\tau_{M}, N(k T)=0, N((k+1) T)>0 \\
k T<\tau_{M}<(k+1) T, N(k T)=0, N((k+1) T)>0
\end{array}\right.
$$

where $\tau_{M}$ denotes the random variable that represents the time from the installation of the system to the first time that the deterioration exceeds the preventive threshold $M$. After some calculations, the expected time to a system replacement for this inspection model is given by (see Appendix Appendix A for proof)

$$
E\left[\tau_{r}\right]=\sum_{k=0}^{\infty}(k+1) T\left(R_{1}(k, T) 1_{\left\{M_{s} \leq M\right\}}+R_{2}(k, T) 1_{\left\{M_{s}>M\right\}}\right)
$$

where $R_{1}(k, T)$ and $R_{2}(k, T)$ are given by

$$
\begin{aligned}
R_{1}(k, T) & =\bar{F}_{1}(k T) \bar{F}_{\tau_{M_{s}}}(k T)-\bar{F}_{1}((k+1) T) \bar{F}_{\tau_{M_{s}}}((k+1) T)+\bar{F}_{2}(k T) \int_{0}^{k T} a(u) \bar{F}_{\tau_{M}-\tau_{M_{s}}}(k T-u) d u \\
& -\bar{F}_{2}((k+1) T) \int_{0}^{(k+1) T} a(u) \bar{F}_{\tau_{M}-\tau_{M_{s}}}((k+1) T-u) d u \\
R_{2}(k, T) & =\bar{F}_{1}(k T) \bar{F}_{\tau_{M}}(k T)-\bar{F}_{1}((k+1) T) \bar{F}_{\tau_{M}}((k+1) T)
\end{aligned}
$$

and $a(t)$ is given by

$$
a(t)=\frac{f_{\tau_{M_{s}}}(t) \bar{F}_{1}(t)}{\bar{F}_{2}(t)}, \quad t \geq 0,
$$


$\bar{F}_{1}$ and $\bar{F}_{2}$ are given by $(9), \bar{F}_{\tau_{M_{s}}}\left(\bar{F}_{\tau_{M}}\right)$ can be obtained using $(2), f_{\tau_{M_{s}}}$ is given by $(3)$ and $\bar{F}_{\tau_{M}-\tau_{M_{s}}}$ can be obtained using (4) and approximated using (6).

Under the considered periodic inspection/replacement policy, a preventive maintenance is performed if the inspection detects that the deterioration of the system exceeds the value $M$ but the system is still working. Thus the probability of a preventive maintenance in a replacement cycle is given by (see Appendix Appendix B for proof)

$$
P_{p}(T, M)= \begin{cases}\sum_{k=0}^{\infty} P\left(A_{1}(k, T)\right)+P\left(A_{2}(k, T)\right) & \text { if } M_{s} \leq M \\ \sum_{k=0}^{\infty} P\left(A_{3}(k, T)\right)+P\left(A_{4}(k, T)\right) & \text { if } M_{s}>M\end{cases}
$$

where for $k=0,1,2, \ldots$ and $T>0$, the quantities $P\left(A_{1}(k, T)\right), P\left(A_{2}(k, T)\right), P\left(A_{3}(k, T)\right)$ and $P\left(A_{4}(k, T)\right)$ are respectively given by

$$
\begin{aligned}
& P\left(A_{1}(k, T)\right)=\bar{F}_{2}((k+1) T) \int_{0}^{k T} a(u)\left(\int_{k T-u}^{(k+1) T-u} f_{\tau_{M}-\tau_{M_{s}}}(v) \bar{F}_{\tau_{L}-\tau_{M}}((k+1) T-u-v) d v\right) d u \\
& P\left(A_{2}(k, T)\right)=\bar{F}_{2}((k+1) T) \int_{k T}^{(k+1) T} a(u)\left(\int_{0}^{(k+1) T-u} f_{\tau_{M}-\tau_{M_{s}}}(v) \bar{F}_{\tau_{L}-\tau_{M}}((k+1) T-u-v) d v\right) d u \\
& P\left(A_{3}(k, T)\right)=\bar{F}_{2}((k+1) T) \int_{k T}^{(k+1) T} f_{\tau_{M}}(u)\left(\int_{0}^{(k+1) T-u} f_{\left.\tau_{M_{s}-\tau_{M}}(v) \bar{F}_{\tau_{L}-\tau_{M_{s}}}((k+1) T-u-v) \frac{\bar{F}_{1}(u+v)}{\bar{F}_{2}(u+v)} d v\right) d u}\right. \\
& P\left(A_{4}(k, T)\right)=\bar{F}_{1}((k+1) T) \int_{k T}^{(k+1) T} f_{\tau_{M}}(u) \bar{F}_{\tau_{M_{s}}-\tau_{M}}((k+1) T-u) d u,
\end{aligned}
$$

where $a(t)$ is given by (15), $\bar{F}_{1}$ and $\bar{F}_{2}$ are given by $(9), f_{\tau_{M}}$ can be obtained from (3) and $f_{\tau_{M}-\tau_{M_{s}}}, f_{\tau_{M_{s}}-\tau_{M}}$, $\bar{F}_{\tau_{L}-\tau_{M}}, \bar{F}_{\tau_{L}-\tau_{M_{s}}}$ and $\bar{F}_{\tau_{M_{s}-\tau_{M}}}$ are obtained using (4) and can be approximated using (6) and (7).

Under the considered periodic inspection/replacement policy, if the system fails between inspections, the system remains failed until the next inspection detects the failure. And the expected down time for the system in a replacement cycle is given by (see Appendix Appendix C for proof)

$$
E\left[W_{r}\right]=\sum_{k=0}^{\infty} \int_{k T}^{(k+1) T}\left(P_{d, k, 1}(t) 1_{\left\{M_{s} \leq M\right\}}+P_{d, k, 2}(t) 1_{\left\{M_{s}>M\right\}}\right) d t
$$

where $P_{d, k, 1}$ and $P_{d, k, 2}$ are given by

$$
\begin{aligned}
P_{d, k, 1}(t) & =-\bar{F}_{2}(t)\left(\int_{0}^{t} a(u) \bar{F}_{\tau_{L}-\tau_{M_{s}}}(t-u) d u-\int_{0}^{k T} a(u) \int_{0}^{k T-u} f_{\tau_{M}-\tau_{M_{s}}}(v) \bar{F}_{\tau_{L}-\tau_{M}}(t-u-v) d v d u\right) \\
& +\bar{F}_{2}(k T) \int_{0}^{k T} a(u) \bar{F}_{\tau_{M}-\tau_{M_{s}}}(k T-u) d u-\bar{F}_{1}(t) \bar{F}_{\tau_{M_{s}}}(t)+\bar{F}_{1}(k T) \bar{F}_{\tau_{M_{s}}}(k T),
\end{aligned}
$$

and,

$$
\begin{aligned}
P_{d, k, 2}(t) & =\bar{F}_{1}(k T) \bar{F}_{\tau_{M}}(k T)-\bar{F}_{1}(t) \bar{F}_{\tau_{M}}(t)-\bar{F}_{1}(t) \int_{k T}^{t} f_{\tau_{M}}(u) \bar{F}_{\tau_{M_{s}}-\tau_{M}}(t-u) d u \\
& -\bar{F}_{2}(t) \int_{k T}^{t} f_{\tau_{M}}(u)\left(\int_{0}^{t-u} f_{\tau_{M_{s}}-\tau_{M}}(v) \frac{\bar{F}_{1}(u+v)}{\bar{F}_{2}(u+v)} \bar{F}_{\tau_{L}-\tau_{M_{s}}}(t-u-v) d v\right) d u,
\end{aligned}
$$


where $a(t)$ is given by (15), $\bar{F}_{1}$ and $\bar{F}_{2}$ are given by $(9), \bar{F}_{\tau_{M_{s}}}$ and $\bar{F}_{\tau_{M}}$ are given by $(2)$ and $\bar{F}_{\tau_{M}-\tau_{M_{s}}}$, $\bar{F}_{\tau_{L}-\tau_{M_{s}}}, \bar{F}_{\tau_{L}-\tau_{M}}$ and $\bar{F}_{\tau_{M_{s}}-\tau_{M}}$ are obtained using (4) and can be approximated using (6).

Considering the expected number of inspections during a replacement cycle $E\left[N_{i}\right]$ is given by

$$
E\left[N_{i}\right]=\frac{E\left[\tau_{r}\right]}{T},
$$

where $E\left[\tau_{r}\right]$ is given by (14).

The optimization problem for this maintenance scheme is reduced to find the values $T$ and $M$ that minimize the function $C_{i n s}(T, M)$ given by (12), that is,

$$
C_{\text {ins }}\left(T_{\text {opt }}, M_{\text {opt }}\right)=\inf \left\{C_{\text {ins }}(T, M), \quad T>0, \quad 0<M<L\right\}
$$

\subsubsection{Numerical example}

To illustrate the cost surface and the optimum solution of the P-I/R policy, we use the following data set: $\alpha=\beta=0.1, L=30, M_{s}=20, r_{1}(t)=\lambda_{1}=0.01, r_{2}(t)=\lambda_{2}=0.1, C_{i}=2, C_{p}=50, C_{c}=100$ and $C_{d}=25$. This data set represents a case of high variance in deterioration increment ( $m=\frac{\alpha}{\beta}=1$, $\left.\sigma^{2}=\frac{\alpha}{\beta^{2}}=10\right)$, high intensity to shock failure and non-expensive inspections. The numerical example of

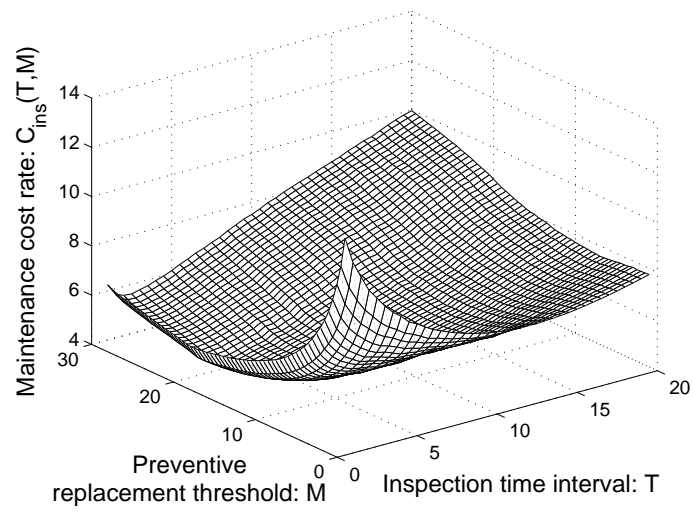

(a) Mesh of expected maintenance cost rate

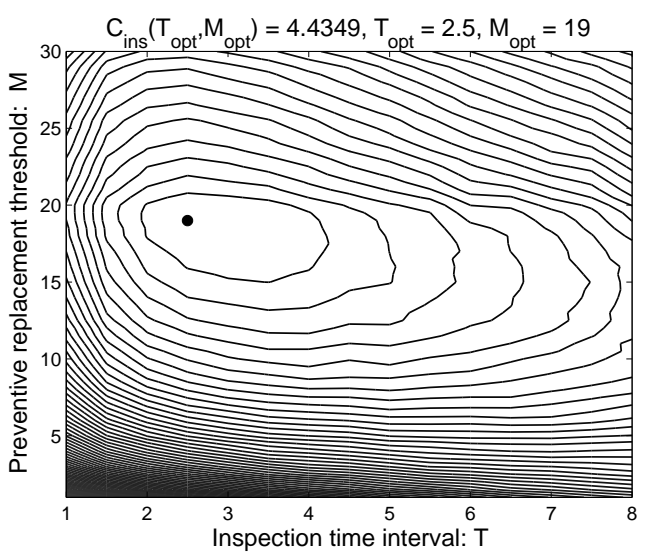

(b) Iso-level of expected maintenance cost rate

Figure 1: An numerical illustration for P-I/R policy

the policy is illustrated in figure 1. Figure 1a illustrates the shape of expected maintenance cost rate of $\mathrm{P}-\mathrm{I} / \mathrm{R}$ policy when the replacement threshold and inter-inspection time interval vary. Figure $1 \mathrm{~b}$ shows that we can obtain an optimal solution under P-I/R policy, and in this case the optimal values of the decision variables (obtained by a classical optimization scheme) are $T_{o p t}=2.5$ and $M_{o p t}=19$ with an optimal cost rate $C_{i n s}\left(T_{o p t}, M_{o p t}\right)=4.4349$. 


\subsection{Block replacement model}

According to the assumptions as in subsection 3.1, a suitable TBM policy for the considered system is the block replacement (B-R) policy [26] because, as in the P-I/R policy the system is not replaced before $T$ even if failed, age-based replacement is not a sensible benchmark policy here. Since preventive replacement decision of the B-R policy is based only on the calendar time of the system and on the knowledge of the statistical information about its lifetime (i.e. regardless of the actual system state), the B-R is considered a "blind" and classical TBM policy. In this paper, this maintenance policy is used as a benchmark to assess the profit of P-I/R policy, as well as the value of condition monitoring information in maintenance decision.

\subsubsection{Block replacement policy}

Under the B-R policy, the system is always replaced at regular time interval $T$. If the system is still running at replacement time, this is a preventive replacement with $\operatorname{cost} C_{p}$; but if the system has already failed, a higher replacement cost is incurred $C_{c}>C_{p}$ and this is regarded as a corrective replacement. In this case there exists a system inactivity time interval after failure, and an additional cost is incurred from the failure time until the replacement time at a cost rate $C_{d}$. The optimal solution of this maintenance policy is to find the optimal values of regular time interval $T_{\text {opt }}$ which minimizes the cost criterion (11).

\subsubsection{Cost model formulation}

Applying (11), the expected cost rate for this maintenance model is given by

$$
C_{b}(T)=\frac{C_{p} \bar{F}_{f}(T)+C_{c} F_{f}(T)+C_{d} \int_{0}^{T} F_{f}(t) d t}{T}, \quad T>0,
$$

where $\bar{F}_{f}(t)$ denotes the survival distribution of the time to failure for the system, and $F_{f}(t)=1-\bar{F}_{f}(t)$.

Denoting by $N(t)$ the number of shock failures in $[0, t]$, the survival function of the time to failure is given by

$$
\begin{aligned}
\bar{F}_{f}(t) & =P(X(t)<L, N(t)=0) \\
& =P\left(X(t) \leq M_{s}, N(t)=0\right)+P\left(M_{s}<X(t)<L, N(t)=0\right) \\
& =\bar{F}_{1}(t) \bar{F}_{\tau_{M_{s}}}(t)+\int_{0}^{t} f_{\tau_{M_{s}}}(u) \bar{F}_{1}(u) \bar{F}_{\tau_{L}-\tau_{M_{s}}}(t-u) \exp \left\{-\int_{u}^{t} r_{2}(v) d v\right\} \\
& =\bar{F}_{1}(t) \bar{F}_{\tau_{M_{s}}}(t)+\bar{F}_{2}(t) \int_{0}^{t} a(u) \bar{F}_{\tau_{L}-\tau_{M_{s}}}(t-u) d u, \quad t \geq 0,
\end{aligned}
$$

where $F_{\tau_{M_{s}}}$ (resp. $f_{\tau_{M_{s}}}$ ) denotes the distribution (resp. density) function of the hitting time for the deterioration level $M_{s}$ obtained using (2) and (resp. (3)), $\bar{F}_{1}$ and $\bar{F}_{2}$ are the survival functions given by (9), $F_{\tau_{L}-\tau_{M_{s}}}$ denotes the distribution of the random variable $\tau_{L}-\tau_{M_{s}}$ with distribution obtained using (4) and the function $a(t)$ is given by (15). 
The optimization problem for this maintenance strategy is to find a value of $T$ that minimizes $C_{b}(T)$ given by (20); in other words, to find a value $T_{\text {opt }}$ such that minimizes the cost function given in (20), that is,

$$
C_{b}\left(T_{o p t}\right)=\inf \left\{C_{b}(T), \quad T>0\right\}
$$

\section{Numerical examples and discussions}

This section aims to investigate the advantage of $\mathrm{P}-\mathrm{I} / \mathrm{R}$ policy by comparing with the benchmark B-R policy through the concrete numerical examples. Firstly, we shall analyze the influence of the maintenance action costs on the optimal values of the decision variables on the evolution of the optimal expected cost rate of both maintenance policies. Secondly, for different possible characteristics of system, the evolution of the optimal expected cost rate as a function of inspection cost is studied. Furthermore, a comparison with the cost incurred by the block replacement strategy allows weighing the benefit of the information returned by inspections against its cost.

\subsection{Sensitivity to the maintenance actions costs}

In order to analyze the sensitivity of the maintenance policies to the maintenance actions costs, we vary one of maintenance costs (i.e. inspection cost, preventive replacement cost or the cost rate for the system inactivity), fix the other costs and investigate the evolution of the optimal values of the decision variables and of the optimal expected maintenance cost rate. It is noted that the corrective replacement cost doesn't influence on the difference between both P-I/R policy and B-R policy, so we can fix the corrective replacement $\operatorname{cost} C_{c}=100$. The practical constraint of maintenance costs $C_{i}<C_{p}<C_{c}$ leads us to consider three following case studies:

1. Case 1. Varied preventive replacement cost and the other costs fixed: $C_{i}=2, C_{p}=2: 1: 100$, $C_{c}=100$ and $C_{d}=25$.

2. Case 2. Varied inspection cost and the other costs fixed: $C_{i}=2: 1: 90, C_{p}=90, C_{c}=100$ and $C_{d}=25$.

3. Case 3. Varied cost rate of system inactivity and the other costs fixed: $C_{i}=5, C_{p}=50, C_{c}=100$ and $C_{d}=5: 1: 150$.

These case studies are applied to the system defined by the data set: $\alpha=\beta=0.1, L=30, M_{s}=20$, $r_{1}(t)=\lambda_{1}=0.01, r_{2}(t)=\lambda_{2}=0.1$. This is the same system described in the numerical example for P-I/R policy. Note that to have the simpler case study, we choose the intensities to shock failures as the constants, but the developments above allow describing the shock rate as any law. 


\subsubsection{Sensitivity to preventive replacement cost: case 1}

To have an unbiased study of the influence of preventive replacement cost on the evolution of the optimal decision variables and of the optimal expected cost rate for both maintenance policies, we choose the non-expensive inspections and the intermediate cost rate for system inactivity, and vary the preventive replacement cost from inspection cost to corrective replacement cost with the cost step equal to 1 . The results for this case are showed as in figure 2 .
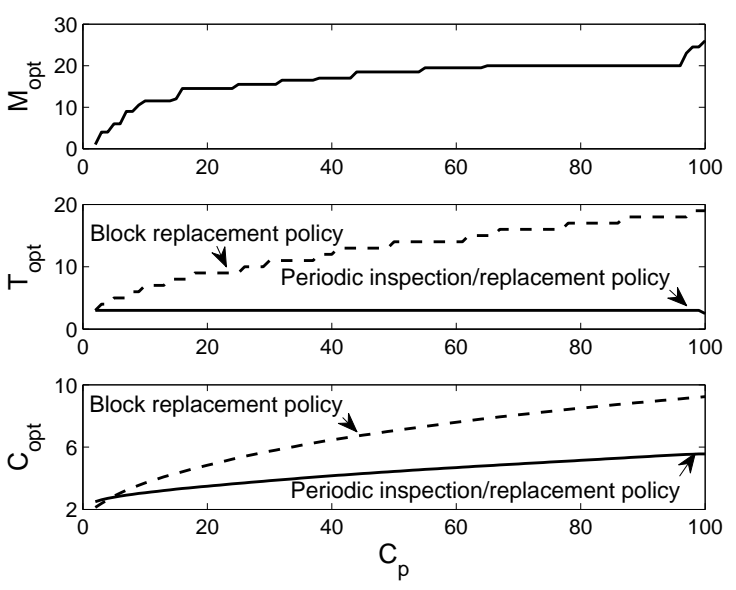

Figure 2: Case 1 - varied preventive replacement cost

The figure shows that the optimal values of inter-inspection interval of $\mathrm{P}-\mathrm{I} / \mathrm{R}$ policy are almost constant against the preventive replacement cost, while the optimal value of preventive replacement threshold in $\mathrm{P}-\mathrm{I} / \mathrm{R}$ policy and the one of regular replacement interval in B-R policy increase with the increasing of preventive replacement cost. This means that few preventive replacements are performed when their cost is high and this also reflects the fundamental difference in the preventive replacement decision between the two considered policies: condition-based replacement for P-I/R policy and time-based replacement for B-R policy. In this case the $\mathrm{P}-\mathrm{I} / \mathrm{R}$ policy is almost always more profitable than B-R policy.

\subsubsection{Sensitivity to inspection cost: case 2}

For this case study, we set the preventive replacement at the high cost, the intermediate cost rate for system inactivity and vary the value inspection cost from a very low cost to preventive replacement cost with the cost step equals 1 . The evolutions of the optimal decision variables and of the optimal expected cost rate for both $\mathrm{P}-\mathrm{I} / \mathrm{R}$ policy and B-R policy for this case study are illustrated in figure 3 .

Obviously the B-R policy does not depend on inspection cost. Not surprisingly, for the P-I/R policy, the optimal value of inter-inspection interval (resp. of the preventive replacement threshold) tends to increase (resp. decrease) when the inspection cost increases. The increasing inter-inspection interval seeks to avoid 

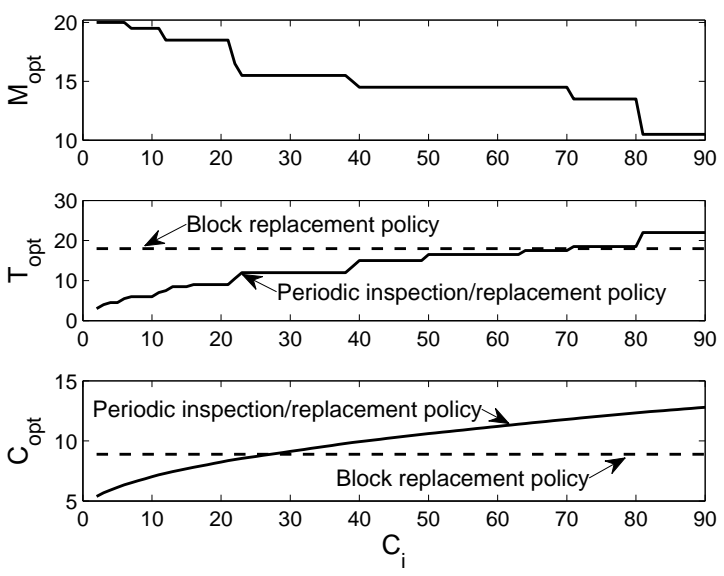

Figure 3: Case 2 - varied inspection cost

more frequent inspections, while the decrease of the value of preventive replacement threshold will limit the probability of system failure. A balance between the frequency of inspections and the preventive replacement threshold may provide an attractive saving in the total maintenance cost. We can also note that the P-I/R policy becomes less advantageous compared to B-R policy when the inspection cost increases. This can be explained by the fact that when the inspection cost is high, the optimal inter-inspection interval is set at a high value, while the optimal preventive replacement threshold is set at a small value (see figure 3). This means that the probability of a replacement at the first inspection is almost 1 , thus the optimal P-I/R policy turns into a B-R policy. However, in this case it is incurred not only the cost to system replacement and system downtime as in the B-R policy, but also an inspection cost to monitor the system state, hence $\mathrm{P}-\mathrm{I} / \mathrm{R}$ policy is less profitable against the B-R policy. When the inspection cost is low, the optimal policy corresponds to a high replacement threshold and involves many inspections in order to monitor precisely the system state so as to trigger a preventive replacement only when necessary and to avoid system unavailability.

\subsubsection{Sensitivity to the cost rate of system inactivity: case 3}

Since this case study concentrates on the analysis of the impact of the system inactivity cost rate on the evolution of the optimal values of the decision variables and of total maintenance cost rate, we set a low cost for inspections, an intermediate cost for preventive replacement (so that here these costs do not influence so much on the results), and vary the cost rate of system inactivity from a very low value to a very high value. Figure 4 reports the result of this analysis.

We can remark that the optimal inter-inspection interval of P-I/R policy, as well as the optimal replacement time of B-R policy decrease when the system inactivity cost rate increases in order to cut short the downtime of system. When the system inactivity cost rate is low, the optimal values of inter-inspection interval in $\mathrm{P}-\mathrm{I} / \mathrm{R}$ policy and of periodic replacement time in B-R policy are high, which means that the 

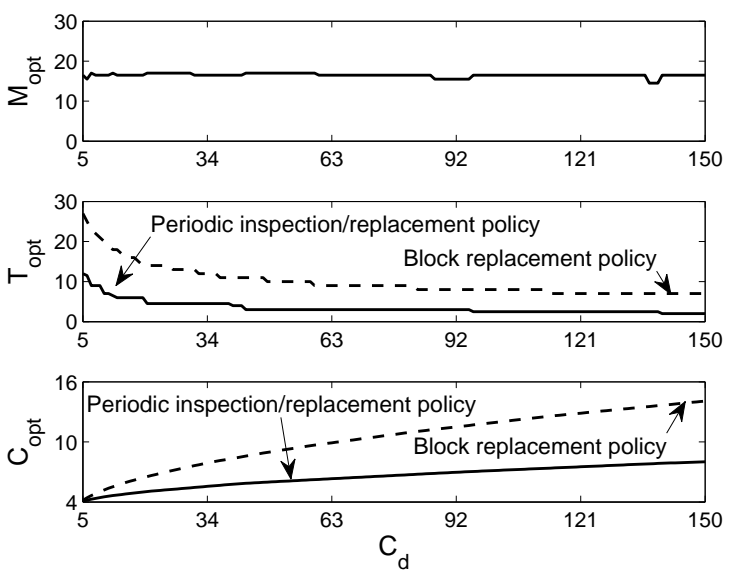

Figure 4: Case 3 - varied cost rate of system inactivity

optimal policies accept a longer downtime interval. The optimal value of preventive replacement does not vary a lot with the system inactivity $\operatorname{cost}$ rate $C_{d}$; the small observed variations are due to the flatness of the cost curve around the optimal value of $M$, and do not correspond to significant cost variation. In this configuration, the most sensitive decision variable is the optimal inter-inspection interval. We see that the P-I/R policy can lead to substantial savings in maintenance cost compared to B-R policy when the system inactivity cost rate is high. This shows that the P-I/R policy can avoid effectively a long system downtime thanks to the more frequent inspections.

These last analyses show that the P-I/R policy in a more general way can adapt quite well to different maintenance costs configurations (inspection cost, preventive replacement cost, system inactivity cost rate). They also provide some indicators on maintenance costs for choosing a suitable maintenance structure according to maintenance costs, i.e. in the case of expensive inspections, a B-R policy could be used instead of the $\mathrm{P}-\mathrm{I} / \mathrm{R}$ policy; for the other cases the $\mathrm{P}-\mathrm{I} / \mathrm{R}$ policy is more interest.

\subsection{Sensitivity to the system dynamic behaviors}

This subsection concentrates on assessing the value of the condition monitoring information for the maintenance decision-making. The nature of the maintenance policy structures, as well as the numerical illustrations in the subsection above show that the inspection cost is the principal impact which causes the different in the performance of both P-I/R policy and B-R policy. Moreover a question arises naturally that under which conditions of system behavior, the P-I/R can replace with profit a more classical B-R policy. Therefore, analogously to the numerical procedure showed by Huynh et al. in [27] and [28], we vary the inspection cost $C_{i}$ and investigate the corresponding evolution of the optimal long run expected maintenance cost rate of P-I/R policy under different possible system characteristics. The comparison of 
this cost to the cost incurred by the block replacement policy allows weighing the benefit of the condition monitoring information returned by inspections against its cost.

We are interested in the impact of degradation variance and of failure rate due to shocks on the optimal maintenance cost rate, so four cases of system behaviors are considered:

1. Case 1. Low variance in deterioration increment and low intensity to shock failures: $\alpha=\beta=1$ $\left(\sigma^{2}=1\right), r_{1}(t)=\lambda_{1}=0.001, r_{2}(t)=\lambda_{2}=0.01$

2. Case 2. High variance in deterioration increment and low intensity to shock failures: $\alpha=\beta=0.1$ $\left(\sigma^{2}=10\right), r_{1}(t)=\lambda_{1}=0.001, r_{2}(t)=\lambda_{2}=0.01$.

3. Case 3. Low variance in deterioration increment and high intensity to shock failures: $\alpha=\beta=1$ $\left(\sigma^{2}=1\right), r_{1}(t)=\lambda_{1}=0.01, r_{2}(t)=\lambda_{2}=0.1$.

4. Case 4. High variance in deterioration increment and high intensity to shock failures: $\alpha=\beta=0.1$ $\left(\sigma^{2}=10\right), r_{1}(t)=\lambda_{1}=0.01, r_{2}(t)=\lambda_{2}=0.1$.

And the other parameters are $L=30, M_{s}=20, C_{p}=50, C_{c}=100$ and $C_{d}=25$. It is noted that the average deterioration rates are the same for these cases and equal $m=1$. Moreover, to have the simpler case study but without loss of generality, we choose the intensities to shock failures as the constants.

The numerical results of these case studies are represented as in Figure 5. The figures show clearly

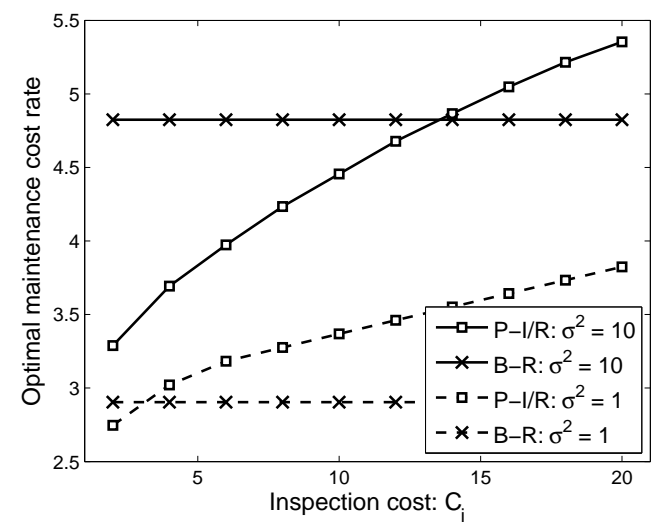

(a) $r_{1}(t)=\lambda_{1}=0.001, r_{2}(t)=\lambda_{2}=0.01$

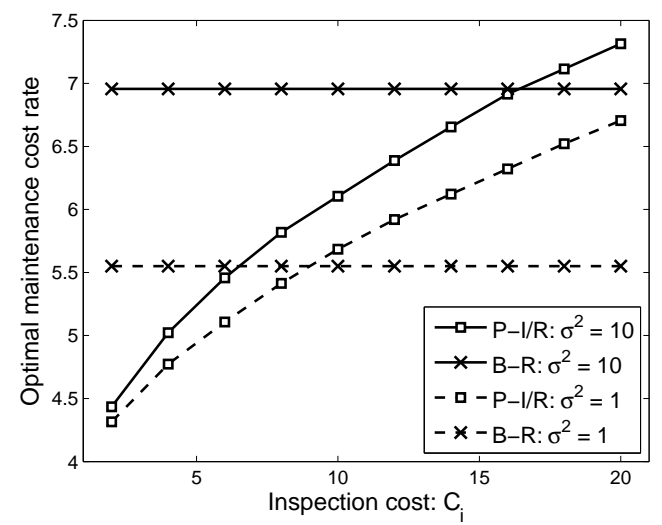

(b) $r_{1}(t)=\lambda_{1}=0.01, r_{2}(t)=\lambda_{2}=0.1$

Figure 5: Comparison on the maintenance cost rates as a function of the inspection costs

that for deterioration processes with high variances in the increments $\left(\sigma^{2}=10\right)$ and/or for traumatic shock process with high intensities to failure $\left(r_{1}(t)=\lambda_{1}=0.01, r_{2}(t)=\lambda_{2}=0.1\right)$, the P-I/R policy may lead to a substantial saving in the maintenance costs. This result can be explained by the following considerations: for a deterioration process with high variance and/or for high frequency of shock failures, an inspection returning the deterioration level and the shock failure speed brings a lot of information on the actual system 
state, and it justifies its cost $C_{i}$. Knowing the deterioration level and the shock failure speed allows the maintenance decision to be adapted to the system state, hence saving maintenance costs when compared to a classical B-R policy.

Generalizing the problem, in comparison with a TBM policy, when the inspection cost is not too high, CBM policy has better capacity to tune the maintenance decision variables (i.e. degradation threshold and inter-inspection time interval) in order to keep the evolution of a system in an optimal zone where maintenance costs optimally balance the different failures and deteriorations costs, so that the profit from the system is maximal. Therefore, it is indeed useful to follow closely the actual evolution of the deterioration path (as well as shock failure rate) to adapt the maintenance decisions to the true state of system, instead of applying the "static" rule of TBM policies based only on the "priori" system lifetime distribution. Obviously, when the inspection cost increases, the CBM loses its interest and become less profitable. That is why the analysis of the maintenance costs savings could be used to justify or not the choice to implement a CBM policy based on condition monitoring and to invest in condition monitoring devices.

\section{Conclusions}

The present paper provides a realistic extension of DTS model by introducing a dependence between the traumatic shock process and degradation process. The analytical cost models of both P-I/R policy and B-R policy have been developed for this more general degradation/failure model. The sensitivity analyses to the different maintenance costs and to different possible characteristics of system provided a thorough quantitative comparison between CBM policy and TBM policy. The results presented in this paper show that we should follow closely the actual evolution of system behaviors to adapt the maintenance decisions to the true system state to improve the maintenance policy performance and decrease its costs. And the analysis of the maintenance costs savings could be used to justify or not the choice to implement a CBM policy based on condition monitoring and to invest in condition monitoring devices.

The perspectives for the problem are essential:

1. For a practical implementation, it is necessary to fit degradation and failure processes to the real data. With the complexity of the proposed degradation/failure model, this is a challenging task. A procedure of parameter estimation (off-line and/or on-line) for this model from the collected data has to be considered in a further work.

2. In the viewpoint of maintenance decision rules, we can take into account the minimal repairs and the non-periodic interventions which are adapted to the observed state of the system, and possible environment covariates.

3. In the viewpoint of quality of the monitoring information, we can consider an imperfect monitoring (non-detectable failures, errors in measurement) or indirect monitoring (covariates). 
The future works will be continued with these two directions.

\section{Appendix A. Proof of equation (14)}

We denote by $\tau_{r}$ the time to a system replacement. Under the assumptions of the model,

$$
\tau_{r}=(k+1) T, \quad k=0,1,2, \ldots
$$

when one of these following events occurs

$$
\begin{aligned}
& B(k, T)=\left\{k T<\tau_{M}<(k+1) T, \quad N((k+1) T)=0\right\} \\
& C(k, T)=\left\{\tau_{M}>(k+1) T, \quad N(k T)=0, \quad N((k+1) T)>0\right\} \\
& D(k, T)=\left\{k T<\tau_{M}<(k+1) T, \quad N(k T)=0, \quad N((k+1) T)>0\right\}
\end{aligned}
$$

Consider the decomposition of the event $B$ into the following exhaustive and mutually exclusive events,

$$
\begin{aligned}
& B_{1}(k, T)=\left\{\tau_{M_{s}}<k T<\tau_{M}<(k+1) T, \quad N((k+1) T)=0\right\} \\
& B_{2}(k, T)=\left\{k T<\tau_{M_{s}}<\tau_{M}<(k+1) T, \quad N((k+1) T)=0\right\} \\
& B_{3}(k, T)=\left\{k T<\tau_{M}<\tau_{M_{s}}<(k+1) T, \quad N((k+1) T)=0\right\} \\
& \left.B_{4}(k, T)=\left\{k T<\tau_{M}<(k+1) T<\tau_{M_{s}}, \quad N((k+1) T)=0\right)\right\}
\end{aligned}
$$

and considering the respective density and distribution function, the probability of the events $B_{i}(k, T)$ where $i=1,2,3,4$, are given by

$$
\begin{aligned}
P\left(B_{1}(k, T)\right) & =\bar{F}_{2}((k+1) T) \int_{0}^{k T} a(u)\left(\int_{(k T-u)}^{(k+1) T-u} f_{\tau_{M}-\tau_{M_{s}}}(v) d v\right) d u \\
P\left(B_{2}(k, T)\right) & =\bar{F}_{2}((k+1) T) \int_{k T}^{(k+1) T} a(u) F_{\tau_{M}-\tau_{M_{s}}}((k+1) T-u) d u \\
P\left(B_{3}(k, T)\right) & =\bar{F}_{2}((k+1) T) \int_{k T}^{(k+1) T} f_{\tau_{M}}(u)\left(\int_{0}^{(k+1) T-u} f_{\tau_{M_{s}}-\tau_{M}}(v) \frac{\bar{F}_{1}(u+v)}{\bar{F}_{2}(u+v)} d v\right) d u \\
P\left(B_{4}(k, T)\right) & =\bar{F}_{1}((k+1) T) \int_{k T}^{(k+1) T} f_{\tau_{M}}(u) \bar{F}_{\tau_{M_{s}}-\tau_{M}}((k+1) T-u) d u
\end{aligned}
$$

where $\bar{F}_{1}$ and $\bar{F}_{2}$ are given by $(9), a(t)$ is given by (15) and $F_{\tau_{M}-\tau_{M_{s}}}\left(F_{\tau_{M_{s}}-\tau_{M}}\right)$ can be obtained using (4). Analogously, we consider the decomposition of the event $C(k, T)$ into the following exhaustive and mutually exclusive events

$$
\begin{aligned}
& C_{1}(k, T)=\left\{\tau_{M_{s}}<k T<(k+1) T<\tau_{M}, \quad N(k T)=0, \quad N((k+1) T)>0\right\}, \\
& C_{2}(k, T)=\left\{k T<\tau_{M_{s}}<(k+1) T<\tau_{M}, \quad N(k T)=0, \quad N((k+1) T)>0\right\}, \\
& C_{3}(k, T)=\left\{(k+1) T<\tau_{M_{s}}<\tau_{M}, \quad N(k T)=0, \quad N((k+1) T)>0\right\}, \\
& C_{4}(k, T)=\left\{(k+1) T<\tau_{M}<\tau_{M_{s}}, \quad N(k T)=0, \quad N((k+1) T)>0\right\},
\end{aligned}
$$


and considering the respective density and distribution functions, the probabilities of the partition of $C$ is given by

$$
\begin{aligned}
P\left(C_{1}(k, T)\right) & =\left(\bar{F}_{2}(k T)-\bar{F}_{2}((k+1) T)\right) \int_{0}^{k T} a(u) \bar{F}_{\tau_{M}-\tau_{M_{s}}}((k+1) T-u) d u \\
P\left(C_{2}(k, T)\right) & =\bar{F}_{1}(k T) \int_{k T}^{(k+1) T} f_{\tau_{M_{s}}}(u) \bar{F}_{\tau_{M}-\tau_{M_{s}}}((k+1) T-u) d u \\
& -\bar{F}_{2}((k+1) T) \int_{k T}^{(k+1) T} a(u) \bar{F}_{\tau_{M}-\tau_{M_{s}}}((k+1) T-u) d u \\
P\left(C_{3}(k, T)\right) & =\bar{F}_{\tau_{M_{s}}}((k+1) T)\left(\bar{F}_{1}(k T)-\bar{F}_{1}((k+1) T)\right) \\
P\left(C_{4}(k, T)\right) & =\bar{F}_{\tau_{M}}((k+1) T)\left(\bar{F}_{1}(k T)-\bar{F}_{1}((k+1) T)\right),
\end{aligned}
$$

where $\bar{F}_{1}$ and $\bar{F}_{2}$ are given by (9), $a(t)$ is given by $(15), F_{\tau_{M_{s}}}\left(F_{\tau_{M}}\right)$ can be obtained using $(2)$ and $\bar{F}_{\tau_{M}-\tau_{M_{s}}}$ using (4). And finally the decomposition of the even $D(k, T)$ into four events $D_{1}(k, T), D_{2}(k, T), D_{3}(k, T)$ and $D_{4}(k, T)$ given by

$$
\begin{aligned}
& D_{1}(k, T)=\left\{\tau_{M_{s}}<k T<\tau_{M}<(k+1) T, N(k T)=0, N((k+1) T)>0\right\} \\
& D_{2}(k, T)=\left\{k T<\tau_{M_{s}}<\tau_{M}<(k+1) T, N(k T)=0, N((k+1) T)>0\right\} \\
& D_{3}(k, T)=\left\{k T<\tau_{M}<\tau_{M_{s}}<(k+1) T, N(k T)=0, N((k+1) T)>0\right\} \\
& D_{4}(k, T)=\left\{k T<\tau_{M}<(k+1) T<\tau_{M_{s}}, N(k T)=0, N((k+1) T)>0\right\}
\end{aligned}
$$

with corresponding probabilities given by

$$
\begin{aligned}
& P\left(D_{1}(k, T)\right)=\left(\bar{F}_{2}(k T)-\bar{F}_{2}((k+1) T)\right) \int_{0}^{k T} a(u)\left(\int_{k T-u}^{(k+1) T-u} f_{\tau_{M}-\tau_{M_{s}}}(v) d v\right) d u \\
& P\left(D_{2}(k, T)\right)=\bar{F}_{1}(k T) \int_{k T}^{(k+1) T} f_{\tau_{M_{s}}}(u) F_{\tau_{M}-\tau_{M_{s}}}((k+1) T-u) \\
& \text { - } \bar{F}_{2}((k+1) T) \int_{k T}^{(k+1) T} a(u) F_{\tau_{M}-\tau_{M_{s}}}((k+1) T-u) d u
\end{aligned}
$$

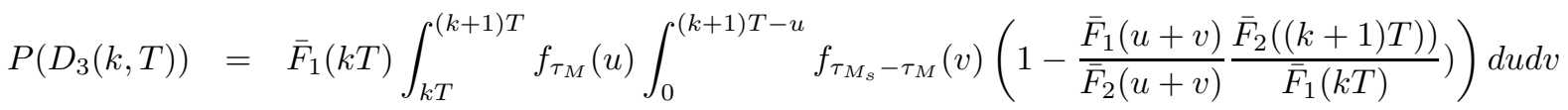

$$
\begin{aligned}
& P\left(D_{4}(k, T)\right)=\left(\bar{F}_{1}(k T)-\bar{F}_{1}((k+1) T)\right) \int_{k T}^{(k+1) T} f_{\tau_{M}}(u) \bar{F}_{\tau_{M_{s}}-\tau_{M}}((k+1) T-u) d u,
\end{aligned}
$$

where $\bar{F}_{1}$ and $\bar{F}_{2}$ are given by $(9), a(t)$ is given by (15), $f_{\tau_{M_{s}}}$ and $f_{\tau_{M}}$ can be obtained using (3) and $\bar{F}_{\tau_{M}-\tau_{M_{s}}}$ using (4).

Let $R_{1}(k, T)$ be the probability of a replacement at time $(k+1) T$ when $M_{s} \leq M$. Considering the 
previous probabilities, one obtains that

$$
\begin{aligned}
R_{1}(k, T) & =\sum_{i=1}^{2}\left(P\left(B_{i}(k, T)\right)+P\left(D_{i}(k, T)\right)\right)+\sum_{i=1}^{3} P\left(C_{i}(k, T)\right) \\
& =\bar{F}_{1}(k T) \bar{F}_{\tau_{M_{s}}}(k T)-\bar{F}_{1}((k+1) T) \bar{F}_{\tau_{M_{s}}}((k+1) T)+\bar{F}_{2}(k T) \int_{0}^{k T} a(u) \bar{F}_{\tau_{M}-\tau_{M_{s}}}(k T-u) d u \\
& -\bar{F}_{2}((k+1) T) \int_{0}^{(k+1) T} a(u) \bar{F}_{\tau_{M}-\tau_{M_{s}}}((k+1) T-u) d u .
\end{aligned}
$$

Analogously, let $R_{2}(k, T)$ be the probability of a replacement at time $(k+1) T$ when $M_{s} \geq M$. The expression $R_{2}(k, T)$ is given by

$$
\begin{aligned}
R_{2}(k, T) & =\sum_{i=3}^{4}\left(P\left(B_{i}\right)+P\left(D_{i}\right)\right)+P\left(C_{4}\right) \\
& =\bar{F}_{1}(k T) \bar{F}_{\tau_{M}}(k T)-\bar{F}_{1}((k+1) T) \bar{F}_{\tau_{M}}((k+1) T)
\end{aligned}
$$

and the result holds.

\section{Appendix B. Proof of equation (16)}

For $M_{s}<M$, a preventive maintenance is performed in the following cases

$$
\begin{aligned}
& A_{1}(k, T)=\left\{\tau_{M_{s}}<k T<\tau_{M}<(k+1) T<\tau_{L}, \quad N((k+1) T)=0\right\} \\
& A_{2}(k, T)=\left\{k T<\tau_{M_{s}}<\tau_{M}<(k+1) T<\tau_{L}, \quad N((k+1) T)=0\right\}
\end{aligned}
$$

and the probabilities of these events are given by

$$
\begin{aligned}
& P\left(A_{1}(k, T)\right)=\bar{F}_{2}((k+1) T) \int_{0}^{k T} a(u)\left(\int_{k T-u}^{(k+1) T-u} f_{\tau_{M}-\tau_{M_{s}}}(v) \bar{F}_{\tau_{L}-\tau_{M}}((k+1) T-u-v) d v\right) d u \\
& P\left(A_{2}(k, T)\right)=\bar{F}_{2}((k+1) T) \int_{k T}^{(k+1) T} a(u)\left(\int_{0}^{(k+1) T-u} f_{\tau_{M}-\tau_{M_{s}}}(v) \bar{F}_{\tau_{L}-\tau_{M}}((k+1) T-u-v) d v\right) d u,
\end{aligned}
$$

where $\bar{F}_{1}$ and $\bar{F}_{2}$ are given by $(9), a(t)$ is given by $(15), F_{\tau_{M}-\tau_{M_{s}}}\left(f_{\tau_{M}-\tau_{M_{s}}}\right)$ and $\bar{F}_{\tau_{L}-\tau_{M}}$ can be obtained using (4).

Analogously, for $M_{s}>M$, a preventive maintenance is performed in the following cases

$$
\begin{aligned}
& A_{3}(k, T)=\left\{k T<\tau_{M}<\tau_{M_{s}}<(k+1) T<\tau_{L}, \quad N((k+1) T)=0\right\} \\
& A_{4}(k, T)=\left\{k T<\tau_{M}<(k+1) T<\tau_{M_{s}}, \quad N((k+1) T)=0\right\}
\end{aligned}
$$

and the probabilities of these events are given by

$$
\begin{aligned}
& P\left(A_{3}(k, T)\right)=\bar{F}_{2}((k+1) T) \int_{K T}^{(k+1) T} f_{\tau_{M}}(u)\left(\int_{0}^{(k+1) T-u} f_{\tau_{M_{s}}-\tau_{M}}(v) \bar{F}_{\tau_{L}-\tau_{M_{s}}}((k+1) T-u-v) \frac{\bar{F}_{1}(u+v)}{\bar{F}_{2}(u+v)} d v\right) d u, \\
& P\left(A_{4}(k, T)\right)=\bar{F}_{1}((k+1) T) \int_{k T}^{(k+1) T} f_{\tau_{M}}(u) \bar{F}_{\tau_{M_{s}}-\tau_{M}}((k+1) T-u) d u
\end{aligned}
$$


where $\bar{F}_{1}$ and $\bar{F}_{2}$ are given by (9), $a(t)$ is given by (15), $F_{\tau_{M_{s}}-\tau_{M}}\left(f_{\tau_{M_{s}}-\tau_{M}}\right)$ and $\bar{F}_{\tau_{L}-\tau_{M}}$ can be obtained using (4) and $f_{\tau_{M}}$ using (3).

\section{Appendix C. Proof of equation (17)}

Let $P_{d, k}(t)$ be the probability that the system is down at time $t$ with $k T \leq t<(k+1) T$ in a replacement cycle. The expected time that the system is down in a replacement cycle is given by

$$
\sum_{k=0}^{\infty} \int_{k T}^{(k+1) T} P_{d, k}(t) d t=\sum_{k=0}^{\infty} \int_{k T}^{(k+1) T} P\left(E_{t}(k, T)\right)+P\left(F_{t}(k, T)\right)+P\left(G_{t}(k, T)\right) d t
$$

where

$$
\begin{aligned}
E_{t}(k, T) & =\left\{k T<\tau_{M}<\tau_{L}<t, N(t)=0\right\} \\
F_{t}(k, T) & =\left\{k T<\tau_{M}, t<\tau_{L}, N(k T)=0, N(t)>0\right\} \\
G_{t}(k, T) & =\left\{k T<\tau_{M}<\tau_{L}<t, N(k T)=0, N(t)>0\right\} .
\end{aligned}
$$

for $k=0,1,2, \ldots, T>0$ and $k T \leq t<(k+1) T$. Now, we calculate the probability of each event. Notice that

$$
E_{t}(k, T)=\underbrace{\left\{E_{t}(k, T), \tau_{M_{s}}<k T\right\}}_{E_{t, 1}(k, T)} \cup \underbrace{\left\{E_{t}(k, T), k T<\tau_{M_{s}}<\tau_{M}\right\}}_{E_{t, 2}(k, T)} \cup \underbrace{\left\{E_{t}(k, T), k T<\tau_{M}<\tau_{M_{s}}<\tau_{L}\right\}}_{E_{t, 3}(k, T)},
$$

and the probabilities of the events $E_{t, 1}(k, T), E_{t, 2}(k, T)$ and $E_{t, 3}(k, T)$ are given by

$$
\begin{aligned}
P\left(E_{t, 1}(k, T)\right) & =\bar{F}_{2}(t) \int_{0}^{k T} a(u)\left(\int_{k T-u}^{t-u} f_{\tau_{M}-\tau_{M_{s}}}(v) F_{\tau_{L}-\tau_{M}}(t-u-v) d v\right) d u \\
P\left(E_{t, 2}(k, T)\right) & =\bar{F}_{2}(t) \int_{k T}^{t} a(u)\left(\int_{0}^{t-u} f_{\tau_{M}-\tau_{M_{s}}}(v) F_{\tau_{L}-\tau_{M}}(t-u-v) d v\right) d u \\
P\left(E_{t, 3}(k, T)\right) & =\bar{F}_{2}(t) \int_{k T}^{t} f_{\tau_{M}}(u)\left(\int_{0}^{t-u} f_{\tau_{M_{s}}-\tau_{M}}(v) F_{\tau_{L}-\tau_{M_{s}}}(t-u-v) \frac{\bar{F}_{1}(u+v)}{\bar{F}_{2}(u+v)} d v\right) d u
\end{aligned}
$$

for $k=0,1,2, \ldots, T>0, k T \leq t<(k+1) T$ where $\bar{F}_{1}$ and $\bar{F}_{2}$ are given by (9), function $a(t)$ is given by (15) and $f_{\tau_{M}-\tau_{M_{s}}}, f_{\tau_{M_{s}}-\tau_{M}}, F_{\tau_{L}-\tau_{M}}$ and $F_{\tau_{L}-\tau_{M_{s}}}$ using (4).

Analogously, the event $F_{t}(k, T)$ can be expressed as follows

$$
\begin{aligned}
& F_{t}(k, T)=\underbrace{\left\{F_{t}(k, T), \tau_{M}<t, \tau_{M_{s}}<k T\right\}}_{F_{t, 1}(k, T)} \cup \underbrace{\left\{F_{t}(k, T), \tau_{M}<t, k T<\tau_{M_{s}}<\tau_{M}\right\}}_{F_{t, 2}(k, T)} \cup \underbrace{\left\{F_{t}(k, T), \tau_{M}<\tau_{M_{s}}<t\right\}}_{F_{t, 3}(k, T)} \\
& \cup \underbrace{\left\{F_{t}(k, T), \tau_{M}<t<\tau_{M_{s}}\right\}}_{F_{t, 4}(k, T)} \cup \underbrace{\left\{F_{t}(k, T), \tau_{M_{s}}<k T, t<\tau_{M}\right\}}_{F_{t, 5}(k, T)} \cup \underbrace{\left\{F_{t}(k, T), t<\tau_{M}, k T<\tau_{M_{s}}<t\right\}}_{F_{t, 7}(k, T)} \\
& \cup \underbrace{\left\{F_{t}(k, T), t<\tau_{M_{s}}<\tau_{M}\right\}}_{F_{t, 8}(k, T)} \cup \underbrace{\left\{F_{t}(k, T)\right.}_{\left.F_{t}(k, T), t<\tau_{M}<\tau_{M_{s}}\right\}}
\end{aligned}
$$


with probabilities

$$
\begin{aligned}
& P\left(F_{t, 1}(k, T)\right)=\left(\bar{F}_{2}(k T)-\bar{F}_{2}(t)\right) \int_{0}^{k T} a(u)\left(\int_{k T-u}^{t-u} f_{\tau_{M}-\tau_{M_{s}}}(v) \bar{F}_{\tau_{L}-\tau_{M}}(t-u-v) d v\right) d u \\
& P\left(F_{t, 2}(k, T)\right)=\bar{F}_{1}(k T) \int_{k T}^{t} f_{\tau_{M_{s}}}(u)\left(\int_{0}^{t-u} f_{\tau_{M}-\tau_{M_{s}}}(v) \bar{F}_{\tau_{L}-\tau_{M}}(t-u-v)\left(1-\frac{\bar{F}_{1}(u)}{\bar{F}_{1}(k T)} \frac{\bar{F}_{2}(t)}{\bar{F}_{2}(u)}\right) d v\right) d u \\
& P\left(F_{t, 3}(k, T)\right)=\bar{F}_{1}(k T) \int_{k T}^{t} f_{\tau_{M}}(u)\left(\int_{0}^{t-u} f_{\tau_{M_{s}-\tau_{M}}}(v) \bar{F}_{\tau_{L}-\tau_{M_{s}}}(t-u-v)\left(1-\frac{\bar{F}_{1}(u+v)}{\bar{F}_{1}(k T)} \frac{\bar{F}_{2}(t)}{\bar{F}_{2}(u+v)}\right) d v\right) d u \\
& P\left(F_{t, 4}(k, T)\right)=\left(\bar{F}_{1}(k T)-\bar{F}_{1}(t)\right) \int_{k T}^{t} f_{\tau_{M}}(u) \bar{F}_{\tau_{M_{s}}-\tau_{M}}(t-u) d u \\
& P\left(F_{t, 5}(k, T)\right)=\left(\bar{F}_{2}(k T)-\bar{F}_{2}(t)\right) \int_{0}^{k T} a(u) \bar{F}_{\tau_{M}-\tau_{M_{s}}}(t-u) d u \\
& P\left(F_{t, 6}(k, T)\right)=\bar{F}_{1}(k T) \int_{k T}^{t} f_{\tau_{M_{s}}}(u) \bar{F}_{\tau_{M}-\tau_{M_{s}}}(t-u)\left(1-\frac{\bar{F}_{1}(u)}{\bar{F}_{1}(k T)} \frac{\bar{F}_{2}(t)}{\bar{F}_{2}(u)}\right) d u \\
& P\left(F_{t, 7}(k, T)\right)=\bar{F}_{\tau_{M_{s}}}(t)\left(\bar{F}_{1}(k T)-\bar{F}_{1}(t)\right) \\
& P\left(F_{t, 8}(k, T)\right)=\bar{F}_{\tau_{M}}(t)\left(\bar{F}_{1}(k T)-\bar{F}_{1}(t)\right),
\end{aligned}
$$

where $\bar{F}_{1}$ and $\bar{F}_{2}$ are given by (9), function $a(t)$ is given by $(15), \bar{F}_{\tau_{M}}$ and $\bar{F}_{\tau_{M_{s}}}$ are obtained using (2) and $f_{\tau_{M}-\tau_{M_{s}}}, f_{\tau_{M_{s}}-\tau_{M}}, \bar{F}_{\tau_{L}-\tau_{M}}, \bar{F}_{\tau_{L}-\tau_{M_{s}}}, \bar{F}_{\tau_{M_{s}-\tau_{M}}}, \bar{F}_{\tau_{M}-\tau_{M_{s}}}$ using (4).

Finally, for the event $G_{t}(k, T)$ with $k=0,1,2, \ldots, k T \leq t<k T$ and $T>0$, one considers the following partition

$$
G_{t}(k, T)=\underbrace{\left\{G_{t}(k, T), \tau_{M_{s}}<k T\right\}}_{G_{t, 1}(k, T)} \cup \underbrace{\left\{G_{t}(k, T), k T<\tau_{M_{s}}<\tau_{M}\right\}}_{G_{t, 2}(k, T)} \cup \underbrace{\left\{G_{t}(k, T), \tau_{M}<\tau_{M_{s}}<\tau_{L}\right\}}_{G_{t, 3}(k, T)}
$$

with probabilities given by

$$
\begin{aligned}
& P\left(G_{t, 1}(k, T)\right)=\left(\bar{F}_{2}(k T)-\bar{F}_{2}(t)\right) \int_{0}^{k T} a(u) \int_{k T-u}^{t-u} f_{\tau_{M}-\tau_{M_{s}}}(v) F_{\tau_{L}-\tau_{M}}(t-u-v) d v \\
& P\left(G_{t, 2}(k, T)\right)=\bar{F}_{1}(k T) \int_{k T}^{t} f_{\tau_{M_{s}}}(u)\left(\int_{0}^{t-u} f_{\tau_{M}-\tau_{M_{s}}}(v) F_{\tau_{L}-\tau_{M}}(t-u-v)\left(1-\frac{\bar{F}_{1}(u) \bar{F}_{2}(t)}{\bar{F}_{1}(k T) \bar{F}_{2}(u)}\right) d v\right) d u \\
& P\left(G_{t, 3}(k, T)\right)=\bar{F}_{1}(k T) \int_{k T}^{t} f_{\tau_{M}}(u)\left(\int_{0}^{t-u} f_{\tau_{M_{s}-\tau_{M}}}(v) F_{\tau_{L}-\tau_{M_{s}}}(t-u-v)\left(1-\frac{\bar{F}_{1}(u+v) \bar{F}_{2}(t)}{\bar{F}_{1}(k T) \bar{F}_{2}(u+v)}\right) d v\right) d u,
\end{aligned}
$$

where $\bar{F}_{1}$ and $\bar{F}_{2}$ are given by (9), function $a(t)$ is given by (15), $\bar{f}_{\tau_{M}}$ and $\bar{f}_{\tau_{M_{s}}}$ are obtained using (3) and $f_{\tau_{M}-\tau_{M_{s}}}, f_{\tau_{M_{s}}-\tau_{M}}, F_{\tau_{L}-\tau_{M}}$ and $F_{\tau_{L}-\tau_{M_{s}}}$ using (4).

Hence, for $M>M_{s}$, the probability that the system is down at time $t$ with $k T \leq t<(k+1) T$ is given by

$$
P_{d, k}(t)=\sum_{i=1}^{2}\left(P\left(E_{t, i}(k, T)\right)+P\left(F_{t, i}(k, T)\right)+G_{t, i}(k, T)\right)+\sum_{i=5}^{7} F_{t, i}(k, T)
$$

and, for $M_{s}>M$,

$$
P_{d, k}(t)=E_{t, 3}(k, T)+F_{t, 3}(k, T)+F_{t, 4}(k, T)+F_{t, 8}(k, T)+G_{t, 3}(k, T),
$$

after some calculus, the result holds. 


\section{Acknowledgements}

For K.T. Huynh this work is part of his $\mathrm{PhD}$ research work financially supported by Conseil Régional de Champagne-Ardernne, France.

For I.T. Castro this research was supported by the Ministerio de Ciencia e Innovación, Spain, under grant MTM2009-07634. The work was performed while I.T. Castro was visiting the University of Technology of Troyes in November 2009. She would like to acknowledge the University of Technology of Troyes for making her stay possible.

[1] P.A. Scarf, A framework for condition monitoring and condition based maintenance, Quality Technology \& Quantitative Management 4 (2) (2007) 301-312.

[2] L. Mann, A. Saxena, G.M. Knapp, Statistical-based or condition-based preventive maintenance, Journal of Quality in Maintenance Engineering 1 (1) (1995) 46-59.

[3] K. Bouvard, S. Artus, C. Bérenguer, V. Cocquempot, Maintenance cost study for deteriorating systems: age-replacement policy vs. condition-based maintenance policy, in: SSARS 2008 - Summer Safety and Reliability Seminars, 2008.

[4] H. Wang, A survey of maintenance policies of deteriorating systems, European Journal of Operational Research 139 (3) (2002) 469-489.

[5] M.D. Pandey, X.X. Yuan, J.M. van Noortwijk, Gamma process model for reliability analysis and replacement of aging structural components, in: ICOSSAR 2005, 2005, pp. 2439-2444.

[6] J.M. van Noortwijk, Optimal replacement decisions for structures under stochastic deterioration, in: Proceedings of the Eighth IFIP WG, Vol. 7, 1998, pp. 273-280.

[7] C.T. Barker, M.J. Newby, Optimal non-periodic inspection for a multivariate degradation model, Reliability Engineering \& System Safety 94 (1) (2009) 33-43.

[8] C. Bérenguer, A. Grall, L. Dieulle, M. Roussignol, Maintenance policy for a continuously monitored deteriorating system, Probability in the Engineering and Informational Sciences 17 (02) (2003) 235-250.

[9] A. Grall, L. Dieulle, C. Bérenguer, M. Roussignol, Continuous-time predictive-maintenance scheduling for adeteriorating system, IEEE Transactions on Reliability 51 (2) (2002) 141-150.

[10] N.D. Singpurwalla, Survival in dynamic environments, Statistical Science 10 (1) (1995) 86-103.

[11] E. Deloux, B. Castanier, C. Bérenguer, Predictive maintenance policy for a gradually deteriorating system subject to stress, Reliability Engineering \& System Safety 94 (2) (2009) 418-431.

[12] A. Lehmann, Joint modeling of degradation and failure time data, Journal of Statistical Planning and Inference 139 (5) (2009) 1693-1706.

[13] W. Li, H. Pham, An inspection-maintenance model for systems with multiple competing processes, IEEE Transactions on Reliability 54 (2).

[14] J.M. Van Noortwijk, J.A.M. Van der Weide, M.J. Kallen, M.D. Pandey, Gamma processes and peaks-over-threshold distributions for time-dependent reliability, Reliability Engineering \& System Safety 92 (12) (2007) 1651-1658.

[15] D. Bocchetti, M. Giorgio, M. Guida, G. Pulcini, A competing risk model for the reliability of cylinder liners in marine diesel engines, Reliability Engineering \& System Safety 94 (8) (2009) 1299-1307.

[16] C. Blain, A. Barros, A. Grall, Y. Lefebvre, Modelling of stress corrosion cracking with stochastic processes-application to steam generators, in: Risk, Reliability and Societal Safety, Proceedings of the European Safety and Reliability Conference, 2007, pp. 25-27.

[17] E. Cinlar, E. Osman, Z.P. Bazoant, Stochastic process for extrapolating concrete creep, Journal of the Engineering Mechanics Division 103 (6) (1977) 1069-1088. 
[18] D.M. Frangopol, M.J. Kallen, J.M. Van Noortwijk, Probabilistic models for life-cycle performance of deteriorating structures: review and future directions, Progress in structural engineering and Materials 6 (4) (2004) 197-212.

[19] M. Abdel-Hameed, A gamma wear process, IEEE transactions on Reliability 24 (2) (1975) $152-153$.

[20] J. Van Noortwijk, A survey of the application of gamma processes in maintenance, Reliability Engineering \& System Safety 94 (1) (2009) 2-21.

[21] R.P. Nicolai, Maintenance models for systems subject to measurable deterioration, Ph.D. thesis, Erasmus University Rotterdam - Timbergen Institude, The Netherlands, 2008.

[22] X. Yuan, Stochastic modeling of deterioration in nuclear power plant components, Ph.D. thesis, University of Waterloo, Ontario, Canada, 2007.

[23] B.V. Gnedenko, V.Y. Korolev, Random summation: limit theorems and applications, CRC Press, 1996.

[24] C. Bérenguer, On the mathematical condition-based maintenance modelling for continuously deteriorating systems, International Journal of Materials and Structural Reliability 6 (2) (2008) 133-151.

[25] H.C. Tijms, A first course in stochastic models, John Wiley and Sons, New York, 2003.

[26] M. Rausand, A. Høyland, System reliability theory: models, statistical methods, and applications, Wiley New York, 2004.

[27] K.T. Huynh, A. Barros, C. Bérenguer, I.T. Castro, Value of condition monitoring for a single-unit system subject to competing failures modes due to wear and traumatic events, in: MMR 2009 - Mathematical Methods in Reliability : Theory, Methods, Applications, 2009, pp. 293-297.

[28] K.T. Huynh, A. Barros, C. Bérenguer, I.T. Castro, Value of condition monitoring information for maintenance decisionmaking, in: Proc. Ann. Reliability \& Maintainability Symp, 2010, pp. 543-548. 